ESAIM: PROCEEDINGS, November 2005, Vol.15, 58-74

T. Goudon, E. Sonnendrucker \& D. Talay, Editors

DOI: $10.1051 /$ proc:2005021

\title{
CONSERVATIVE AND ENTROPIC NUMERICAL SCHEMES FOR THE NON HOMOGENEOUS FOKKER-PLANCK-LANDAU EQUATION
}

\author{
Nicolas Crouseilles ${ }^{1}$
}

\begin{abstract}
In this paper, we investigate the approximation of the solution to the Vlasov equation coupled with the Fokker-Planck-Landau collision operator using a phase space grid. On the one hand, the transport is treated by a flux conservative method and the distribution function is reconstructed to to preserve energy and to control numerical oscillations. On the other hand, the collision operators are approximated to preserve the main properties (conservations and decrease of the entropy). Several numerical results are presented in one dimension in space and three dimensions in velocity.
\end{abstract}

Résumé. Dans cet article, on étudie l'approximation de la solution de l'équation de Vlasov couplée avec l'opérateur de collisions de Fokker-Planck-Landau utilisant une grille de l'espace des phases. D'une part, le transport est traité par une méthode à flux conservatif et la fonction de distribution est reconstruite pour préserver l'énergie et contrôler les oscillations numériques. D'autre part, les opérateurs de collisions sont approchés de façon à préserver leurs propriétés fondamentales (lois de conservations et décroissance de l'entropie). Divers résultats numériques sont présentés en dimension un d'espace et dimension trois en vitesse.

Keywords: Landau-Fokker-Planck equation, Vlasov-Poisson system, finite difference methods.

\section{INTRODUCTION}

The evolution of a collisional plasma constituted of different species of particles is commonly described by the Landau or Fokker-Planck-Landau (FPL) equation at the kinetic level (see [5,7-9]). It describes binary collisions between charged particles with long-range Coulomb interactions. The evolution of particles $\alpha$ is given by the distribution function $f_{\alpha}(t, x, v)$, which depends on time $t$, position $x \in \Omega \subset \mathbb{R}^{3}$ and velocity $v \in \mathbb{R}^{3}$. This distribution function is solution to the scaled Fokker-Planck-Landau equation

$$
\frac{\partial f_{\alpha}}{\partial t}+v \cdot \nabla_{x} f_{\alpha}+E \cdot \nabla_{v} f_{\alpha}=\nu \sum_{\beta} Q_{\alpha, \beta}\left(f_{\alpha}, f_{\beta}\right)
$$

where $f_{\beta}$ is the distribution function of particles $\beta, E=E(t, x)$ is the self-consistent electric field which can evolve through the Poisson equation

$$
\nabla_{x} \cdot E(t, x)=\sum_{\beta} \int_{\mathbb{R}^{3}} f_{\beta}(v) d v, \quad E(t, x)=-\nabla_{x} \phi(t, x),
$$

\footnotetext{
${ }^{1}$ Mathématiques pour l'Industrie et la Physique, CNRS UMR 5640, INSA et Université Paul Sabatier - Toulouse 3, 118 route de Narbonne, F-31062 Toulouse cedex 4, France. crouseilles@mip.ups-tlse.fr
} 
where $\phi=\phi(t, x)$ is the electric potential. Finally, $\nu$ is a nonnegative constant related to the collision frequency, $Q_{\alpha, \beta}\left(f_{\alpha}, f_{\beta}\right)$ describes $\alpha-\beta$ collisions and reads

$$
Q_{\alpha, \beta}\left(f_{\alpha}, f_{\beta}\right)=\nabla_{v} \cdot\left(\int_{\mathbb{R}^{3}} \Phi\left(v-v^{\prime}\right)\left(\nabla_{v} f_{\alpha}(v) f_{\beta}\left(v^{\prime}\right)-\nabla_{v^{\prime}} f_{\beta}\left(v^{\prime}\right) f_{\alpha}(v)\right) d v^{\prime}\right)
$$

where $\Phi(v)$ is the $3 \times 3$ matrix

$$
\Phi(v)=\frac{1}{|v|^{3}} S(v)
$$

and

$$
S(v)=|v|^{2} I_{3}-v \otimes v
$$

Many different deterministic numerical schemes have been considered to Fokker-Planck type equations $[2,3,8$, 13,16]. Due to the computational complexity of the equation (essentially caused by the large number of variables and the three-fold collision integral), many papers have been devoted to treat simpler space homogeneous situations (the distribution function $f_{\alpha}$ does not depend on $x$ ) in the isotropic case or for cylindrically symmetric problems. The construction of conservative and entropic schemes for the space homogeneous case has been proposed by Degond and Lucquin-Desreux in [8] and by Buet and Cordier [2]. These schemes are built in such a way that the main physical properties are conserved at a discrete level. Positivity of the solution and discrete entropy inequality are also satisfied.

Most of these methods have proven their efficiency in the homogeneous case, but few results are available in the non homogeneous situation (see $[4,11]$ ). The main goal of this work is to develop a scheme in the $x$ depending case and in the whole $3 \mathrm{D}$ velocity space.

The first step consists in the approximation of the Vlasov equation, which is the left hand side of (1.1). Its numerical resolution is often performed through particle methods (Particle In Cell) where the plasma is approached by a finite number of macro-particles (see [1]). Even if these methods give satisfying results, it is well known that particle methods are noisy. Consequently, methods which discretize the Vlasov equation on a phase space grid have been proposed $[10,17]$. These approaches allow to get an accurate approximation of the distribution function in the phase space, but the non conservation of the energy due to the projection on the grid and interpolation can be an inconvenient for the long time behavior of the solution. We then propose a new scheme using a phase space grid which overcomes these inconvenients: space and velocity derivatives are approximated by a centered finite volume method and an adapted approximation of the electric field $E$ allows us to obtain a numerical scheme that conserves the total energy. As this kind of discretization does not ensure the positivity of the unknown, we introduce slope correctors. The distribution function is reconstructed following the second order PFC method (see $[10,11]$ ). However, when the slope correctors act (to avoid negative values for $f$ ) the total energy is not conserved any more.

Finally, we deal with the approximation of the collision operators $Q_{\alpha, \beta}$ given by (1.3). We first perform some assumptions on the different species of particles to get a simpler collision operator when $\alpha$ differs from $\beta$. Then, we propose a conservative scheme, based on the ideas of [8] to approximate this new operator, where particles are always interacting by Colombian potentials. For $\alpha$ - $\alpha$ interactions, the full Fokker-Planck-Landau collision operator is used. The construction of a conservative and entropic scheme for the general situation has been proposed in [8]. Even if this scheme gives interesting properties, its direct implementation is very expensive in high dimensions. Thus, we adopt the multigrid method used in [3] to reduce the computational cost.

The rest of the paper is organized as follows. In the next section, we draw up the main properties of the solution to the Vlasov-Poisson system coupled with Fokker-Planck-Landau collision operators and formally derive the operator intended to model collisions between different species. We then present in section 3 , a finite volume scheme for the discretization of the Vlasov-Poisson equation. We next propose an approximation of the simplified collision operator and describe the discretization of the full Fokker-Planck-Landau operator. Finally, several numerical results are presented in section 4 to illustrate the efficiency of the method. 


\section{Description of the Kinetic model}

\subsection{Transport equation}

We first briefly recall some classical estimates on the Vlasov-Poisson (1.1)-(1.2) system without collision (i.e. $\nu=0)$ : mass and momentum are preserved with time,

$$
\frac{d}{d t} \int_{\mathbf{R}^{3} \times \mathbf{R}^{3}} f_{\alpha}(t, x, v)\left(\begin{array}{c}
1 \\
v
\end{array}\right) d x d v=0, \quad t \in \mathbb{R}^{+} .
$$

Next, multiplying the Vlasov equation (1.1) (with $\nu=0$ ) by $|v|^{2}$ and performing an integration by parts, we find the conservation of energy for the (1.1)-(1.2) system

$$
\frac{d}{d t}\left(\int_{\mathbf{R}^{3} \times \mathbf{R}^{3}} f_{\alpha}(t, x, v) \frac{|v|^{2}}{2} d x d v+\frac{1}{2} \int_{\mathbf{R}^{3}}|E(t, x)|^{2} d x\right)=0, \quad t \in \mathbb{R}^{+} .
$$

Finally, the Vlasov-Poisson equation (1.1)-(1.2) conserves the kinetic entropy

$$
H(t)=\int_{\mathbf{R}^{3} \times \mathbf{R}^{3}} f_{\alpha}(t) \log \left(f_{\alpha}(t)\right) d x d v=H(0) .
$$

\subsection{Collision operators}

The Fokker-Planck-Landau (FPL) operator is used for the description of binary collisions between charged particles, for which the interaction potential is the long-range Coulomb interaction. In our case, it describes $\alpha-\alpha$ collisions. We recall the expression and some properties of the FPL collision operator

$$
Q_{\alpha, \alpha}\left(f_{\alpha}, f_{\alpha}\right)=\nabla_{v} \cdot\left(\int_{\mathbb{R}^{3}} \Phi\left(v-v^{\prime}\right)\left(\nabla_{v} f_{\alpha}(v) f_{\alpha}\left(v^{\prime}\right)-\nabla_{v^{\prime}} f_{\alpha}\left(v^{\prime}\right) f_{\alpha}(v)\right) d v^{\prime}\right),
$$

where $\Phi(v)$ is the $3 \times 3$ matrix (1.4). The algebraic structure of the FPL operator is similar to the Boltzmann one, this leads to physical properties ; indeed, mass, momentum and energy are conserved

$$
\int_{\mathbb{R}^{3}} Q_{\alpha, \alpha}\left(f_{\alpha}, f_{\alpha}\right)(v),\left(\begin{array}{l}
1 \\
v \\
|v|^{2}
\end{array}\right) d v=0
$$

and the entropy $H(t)$ is decreasing,

$$
\frac{d H}{d t}(t)=\frac{d}{d t} \int_{\mathbf{R}^{3}} f_{\alpha}(t, v) \log \left(f_{\alpha}(t, v)\right) d v \leq 0 .
$$

Finally, the equilibrium state of the FPL operator, i.e. the distribution function $f_{\alpha}$ which satisfies $Q_{\alpha, \alpha}\left(f_{\alpha}, f_{\alpha}\right)=$ 0 , is given by a Maxwellian

$$
\mathcal{M}_{\rho_{\alpha}, u_{\alpha}, T_{\alpha}}(v)=\frac{\rho_{\alpha}}{\left(2 \pi T_{\alpha}\right)^{3 / 2}} \exp \left(-\frac{\left|v-u_{\alpha}\right|^{2}}{2 T_{\alpha}}\right)
$$

where $\rho_{\alpha}$ is the total mass, $u_{\alpha}$ the mean velocity and $T_{\alpha}$ the temperature given by

$$
\rho_{\alpha}=\int_{\mathbb{R}^{3}} f_{\alpha}(v) d v, \quad u_{\alpha}=\frac{1}{\rho_{\alpha}} \int_{\mathbf{R}^{3}} f_{\alpha}(v) v d v, \quad T_{\alpha}=\frac{1}{3 \rho_{\alpha}} \int_{\mathbf{R}^{3}} f_{\alpha}(v)\left|v-u_{\alpha}\right|^{2} d v .
$$

The operator $Q_{\alpha, \beta}$ describes collisions between two different species (for instance electrons and ions) and can be derived from the two species form of the full Landau operator (1.3). If we assume that the temperature $T_{\beta}$ is 
negligeable compared to the temperature $T_{\alpha}$ of species $\alpha(2.2)$, we may consider that the distribution function of species $\beta$ is given by a Dirac measure in velocity

$$
f_{\beta}(t, x, v)=\rho_{\beta}(t, x) \delta_{0}\left(v-u_{\beta}(t, x)\right),
$$

where the density $\rho_{\beta}$ and the mean velocity $u_{\beta}$ are given or satisfy hydrodynamic equations. The so-obtained operator then reads (see [7] for a more physical derivation)

$$
Q_{\alpha, \beta}\left(f_{\alpha}\right)=\rho_{\beta} \nabla_{v} \cdot\left(\Phi\left(v-u_{\beta}\right) \nabla_{v} f_{\alpha}\right)
$$

where $\Phi(v)$ is the $3 \times 3$ matrix given by (1.4).

The so-obtained linear operator (2.4) satisfies some properties which are listed in the following proposition.

Proposition 2.1. The linear collision operator $Q_{\alpha, \beta}\left(f_{\alpha}\right)$ given by (2.4) satisfies

(i) the preservation of mass and energy, i.e.

$$
\int_{\mathbf{R}^{3}} Q_{\alpha, \beta}\left(f_{\alpha}\right)(v) d v=0, \quad \int_{\mathbf{R}^{3}} Q_{\alpha, \beta}\left(f_{\alpha}\right)(v)\left|v-u_{\beta}\right|^{2} d v=0,
$$

(ii) $\operatorname{Ker}\left(Q_{\alpha, \beta}\right)=\left\{f_{\alpha}\left(\left|v-u_{\beta}\right|^{2}\right)\right\}$,

(iii) each convex function $\Psi$ of $f_{\alpha}$ is an entropy for $Q_{\alpha, \beta}$

$$
\frac{d}{d t} \int_{\mathbb{R}^{3}} \Psi\left(f_{\alpha}\right) d v \leq 0 .
$$

For the proof, we refer the reader to [6].

\section{THE NUMERICAL METHOD}

From now, we will only consider one species of particles, then the index $\alpha$ will be dropped and the distribution function will be denoted by $f$.

We first give a finite volume scheme to the Vlasov-Poisson equation and then describe a conservative and entropy decreasing method to the collision operators.

\subsection{Approximation to the Vlasov-Poisson system}

First, we present a discretization of the Vlasov-Poisson equation. Thanks to an approximation based on a finite volume method joint with an adapted approximation of the electric field, we will prove that this scheme preserves the total energy.

For the sake of simplicity, we present the scheme in one dimension in space and velocity, but it can be extended to higher dimensions by applying the same reconstruction in each direction.

We introduce the mesh points $\left(x_{i+1 / 2}\right)_{i \in I}$ and $\left(v_{j+1 / 2}\right)_{j \in \mathbb{Z}}$ of the computational domain $\left[x_{\min }, x_{\max }\right] \times \mathbb{R}$. We will denote by $\Delta x=x_{i+1 / 2}-x_{i-1 / 2}$ and $\Delta v=v_{j+1 / 2}-v_{j-1 / 2}$ the space and velocity steps and by $C_{i, j}=\left[x_{i-1 / 2}, x_{i+1 / 2}\right] \times\left[v_{j-1 / 2}, v_{j+1 / 2}\right]$ the control volume. Finally, $t^{n}=n \Delta t$ is the time discretization.

We assume that $f_{i, j}^{n}$ is an average approximation of $f$ on the control volume $C_{i, j}$ at time $t^{n}$

$$
f_{i, j}^{n}=\frac{1}{\Delta x \Delta v} \int_{C_{i, j}} f\left(t^{n}, x, v\right) d x d v
$$


Then, we approximate the average of $f$ at the time $t^{n+1}$ by integrating the Vlasov equation on a control volume and using a backward Euler scheme in time

$$
\begin{aligned}
f_{i, j}^{n+1}= & f_{i, j}^{n}-\frac{\Delta t}{\Delta x \Delta v} \int_{v_{j-1 / 2}}^{v_{j+1 / 2}} v\left(f\left(t^{n}, x_{i+1 / 2}, v\right)-f^{n}\left(t^{n}, x_{i-1 / 2}, v\right)\right) d v \\
& -\frac{\Delta t}{\Delta x \Delta v} \int_{x_{i-1 / 2}}^{x_{i+1 / 2}} \tilde{E}^{n}(x)\left(f\left(t^{n}, x, v_{j+1 / 2}\right)-f^{n}\left(t^{n}, x, v_{j-1 / 2}\right)\right) d x
\end{aligned}
$$

We have to approach the fluxes at the interface of the control volume $\left[x_{i-1 / 2}, x_{i+1 / 2}\right)$ and $\left[v_{j-1 / 2}, v_{j+1 / 2}\right)$

$$
\frac{1}{\Delta v} \int_{v_{j-1 / 2}}^{v_{j+1 / 2}} v f\left(t^{n}, x_{i+1 / 2}, v\right) d v, \forall i \in I, \quad \frac{1}{\Delta x} \int_{x_{i-1 / 2}}^{x_{i+1 / 2}} \tilde{E}^{n}(x) f\left(t^{n}, x, v_{j+1 / 2}\right) d x, \forall j \in \mathbb{Z},
$$

where $\tilde{E}^{n}(x)$ is an approximation of the electric field deduced from the numerical resolution to the Poisson equation (1.2).

The main step to get an accurate solution is to reconstruct the distribution function in each direction. For that purpose, we fix the velocity $v \in\left[v_{j-1 / 2}, v_{j+1 / 2}\right)$ and consider the function $f_{h}(x, v)$ as an approximation of the distribution function $f\left(t^{n}, x, v\right)$. Thus, the high order approximation $f_{h}(x, v)$ is obtained via a reconstruction by primitive $F_{h}(x, v)$ (where $F_{h}(x, v)$ stands for the primitive of $f_{h}(x, v)$ with respect to $x$ ). We denote by

$$
f_{i}^{n}(v)=\frac{1}{\Delta x} \int_{x_{i-1 / 2}}^{x_{i+1 / 2}} f\left(t^{n}, x, v\right) d x, \text { for } \quad v \in\left[v_{j-1 / 2}, v_{j+1 / 2}\right),
$$

hence,

$$
F_{h}\left(x_{i+1 / 2}, v\right)-F_{h}\left(x_{i-1 / 2}, v\right)=\Delta x f_{i}^{n}(v) .
$$

We present a method of reconstruction allowing to obtain a second order scheme, which preserves the positivity using slope limiters. To this aim, we first build an approximation of the primitive using the stencil $\left\{x_{i-1 / 2}, x_{i+1 / 2}, x_{i+3 / 2}\right\}$ when the velocity $v$ is positive and $\left\{x_{i-3 / 2}, x_{i-1 / 2}, x_{i+1 / 2}\right\}$ when $v$ is negative. Let us assume that $v$ is positive, then we get

$$
F_{h}(x, v)=F_{h}\left(x_{i-1 / 2}, v\right)+\left(x-x_{i-1 / 2}\right) f_{i}^{n}(v)+\frac{\left(x-x_{i-1 / 2}\right)\left(x-x_{i+1 / 2}\right)}{2 \Delta x}\left(f_{i+1}^{n}(v)-f_{i}^{n}(v)\right) .
$$

By differentiation, we obtain a second order accurate approximation of the distribution function on the interval $\left[x_{i-1 / 2}, x_{i+1 / 2}\right)$

$$
f_{h}(x, v)=\frac{\partial F_{h}}{\partial x}(x, v)=f_{i}^{n}(v)+\frac{\left(x-x_{i}\right)}{\Delta x}\left(f_{i+1}^{n}(v)-f_{i}^{n}(v)\right) .
$$

This appears as a second order approximation, which can generate spurious oscillations. We then introduce a slope corrector, ensuring the positivity of the distribution function on the interval $\left[x_{i-1 / 2}, x_{i+1 / 2}\right)$

$$
f_{h}(x, v)=f_{i}^{n}(v)+\epsilon_{i}^{+}(v) \frac{\left(x-x_{i}\right)}{\Delta x}\left(f_{i+1}^{n}(v)-f_{i}^{n}(v)\right)
$$

with

$$
\epsilon_{i}^{+}(v)=\min \left(1,2 f_{i}^{n}(v) /\left(f_{i+1}^{n}(v)-f_{i}^{n}(v)\right)\right), \quad \text { if } \quad\left(f_{i+1}^{n}(v)-f_{i}^{n}(v)\right)>0 .
$$

For a negative velocity $v$, the reconstruction on the interval $\left(x_{i-1 / 2}, x_{i+1 / 2}\right)$ is

$$
f_{h}(x, v)=f_{i}^{n}(v)+\epsilon_{i}^{-}(v) \frac{\left(x-x_{i}\right)}{\Delta x}\left(f_{i}^{n}(v)-f_{i-1}^{n}(v)\right)
$$


with

$$
\epsilon_{i}^{-}(v)=\min \left(1,-2 f_{i}^{n}(v) /\left(f_{i}^{n}(v)-f_{i-1}^{n}(v)\right)\right), \quad \text { if } \quad\left(f_{i}^{n}(v)-f_{i-1}^{n}(v)\right)<0 .
$$

Now, we proceed in the same way to reconstruct $f_{h}$ in the $v$ direction depending on the $\operatorname{sign}$ of $\tilde{E}^{n}(x)$.

When the slope correctors do not occur in the approximation of the velocity derivative (it is the case when the distribution function is sufficiently smooth), we obtain a classical centered scheme.

Thus, from the following approximation of the electric field

$$
\tilde{E}_{i}^{n}=\frac{E_{i}^{n+1}+E_{i}^{n}}{2}
$$

the so-obtained scheme can be written as

$$
f_{i, j}^{n+1}=f_{i, j}^{n}-\frac{\Delta t}{\Delta x \Delta v}\left(\psi_{i+1 / 2, j}^{n}-\psi_{i-1 / 2, j}^{n}\right)-\Delta t\left(\frac{E_{i}^{n+1}+E_{i}^{n}}{2} D_{c, v} f_{i, j}^{n}\right)
$$

where $\psi_{i+1 / 2, j}^{n}=\Delta v v_{j} f_{i+1 / 2, j}^{n}$ and $D_{c, v}$ is the usual second order centered discrete operator

$$
D_{c, v} f_{j}=\frac{f_{j+1}-f_{j-1}}{2 \Delta v}
$$

On the other hand, the electric field at time $t^{n}$ is determined through the following approximation to the Poisson equation

$$
-D_{x}^{\star} E_{i}^{n}=\rho_{i}^{n}-\rho_{\beta}, \quad E_{i}^{n}=-D_{x} \phi_{i}^{n},
$$

where $D_{x}$ is a discrete finite difference operator whereas $D_{x}^{\star}$ stands for its formal adjoint, which represents an approximation of $-\partial_{x}$ (for example, we can consider $D_{x}$ as the usual uncentered discrete operator). Hence, the following equality holds

$$
\left\langle D^{\star} \varphi, f\right\rangle=\langle\varphi, D f\rangle, \text { for all sequences } \varphi, f,
$$

where $\langle$,$\rangle denotes an inner product. Finally, E_{i}^{n+1}$ is a prediction of the electric field at time $t^{n+1}$ obtained from the discretization to the Poisson equation (3.9) and the continuity equation

$$
\rho^{n+1}=\rho_{i}^{n}+\Delta t D_{x}^{\star} J_{i}^{n}
$$

where $J_{i}^{n}$ is an approximation of the current density $j(t, x)$

$$
J_{i}^{n}=\Delta v \sum_{j \in \mathbb{Z}} v_{j} f_{i, j}^{n}
$$

whereas $\rho_{i}^{n}$ is computed iteratively from the initial density.

The approximation of the Vlasov-Poisson equation obtained from this algorithm satisfies the conservation of mass

$$
\Delta x \Delta v \sum_{i, j} f_{i, j}^{n}=\Delta x \Delta v \sum_{i, j} f_{i, j}^{0},
$$

and of total energy

$$
\frac{\Delta x \Delta v}{2} \sum_{i, j}\left|v_{j}\right|^{2} f_{i, j}^{n}+\frac{\Delta x}{2} \sum_{i}\left(E_{i}^{n}\right)^{2}=\frac{\Delta x \Delta v}{2} \sum_{i, j}\left|v_{j}\right|^{2} f_{i, j}^{0}+\frac{\Delta x}{2} \sum_{i}\left(E_{i}^{0}\right)^{2} .
$$

We refer the reader to [6] for the proof. 


\subsection{Approximation to the collision operators}

This section is devoted to the discretization of the collision operators. First, we are going to present an approximation of the linear operator (2.4). We may restrict ourselves to the space homogeneous equation and since $\rho_{\beta}$ and $u_{\beta}$ only play the role of parameters, we set

$$
\rho_{\beta}=1, \quad u_{\beta}=0
$$

Then, we approximate the following equation

$$
\frac{\partial f}{\partial t}=Q_{\alpha, \beta}(f),\left.f\right|_{t=0}=f_{0}(v)
$$

where $Q_{\alpha, \beta}(f)$ is given by $(2.4)$ and $f_{0}$ stands for an initial datum. Let $v_{j}=j \Delta v$ and $j=\left(j_{1}, j_{2}, j_{3}\right) \in \mathbb{Z}^{3}$ be a uniform mesh in $\mathbb{R}^{3}$ and we denote by $f_{j}$ an approximation of $f\left(v_{j}\right)$. We define $D$ as a finite difference operator that approximates the usual gradient operator $\nabla_{v}$ and by $D^{\star}$ its formal adjoint, which represents an approximation of $\left(-\nabla_{v} \cdot\right)$. Then, for any test sequence $\left(\varphi_{j}\right)_{j \in \mathbb{Z}^{3}}$, we set $\left(D \varphi_{j}\right)_{j \in \mathbb{Z}^{3}}$ as a sequence of vectors of $\mathbb{R}^{3}$

$$
D \varphi_{j}=\left(D^{1} \varphi_{j}, D^{2} \varphi_{j}, D^{3} \varphi_{j}\right) \in \mathbb{R}^{3},
$$

where the components $D^{s} \varphi_{j}, s \in\{1,2,3\}$ approximates the partial derivatives $\left(\partial \varphi / \partial v_{s}\right)\left(v_{j}\right)$.

To preserve the property of decreasing entropy at the discrete level, we use the log weak formulation as in [8]. Using the notations introduced above, the operator $Q_{\alpha, \beta}(f)(v)$ can be approximated at $v=v_{j}$ by

$$
Q_{\alpha, \beta}(f)\left(v_{j}\right)=-D^{\star}\left(\frac{1}{\left|v_{j}\right|^{3}} S\left(\tilde{v}_{j}\right) f_{j} D\left(\log f_{j}\right)\right)
$$

where $S\left(\tilde{v}_{j}\right)$ is an approximation at $\tilde{v}_{j}$ to the matrix $S$ given by (1.5) i.e.

$$
S\left(\tilde{v}_{j}\right)=\left|\tilde{v}_{j}\right|^{2} I_{3}-\tilde{v}_{j} \otimes \tilde{v}_{j}
$$

and $\tilde{v}_{j}$ is chosen as a function of $D$ to preserve energy.

Example 3.1. If we choose $D=D_{+}$i.e. the forward uncentered discrete operator, (3.16) implies that $\tilde{v}$ must be chosen as follows

$$
\tilde{v}_{j}^{s}=\frac{1}{2}\left(v_{j}^{s}+v_{j+e_{s}}^{s}\right), \quad s \in\{1,2,3\} .
$$

The choice of $D=D_{-}$leads to the following $\tilde{v}$

$$
\tilde{v}_{j}^{s}=\frac{1}{2}\left(v_{j}^{s}+v_{j-e_{s}}^{s}\right), \quad s \in\{1,2,3\} .
$$

Finally, if $D=D_{c}$ i.e. the centered discrete operator, (3.16) gives for $\tilde{v}$

$$
\tilde{v}_{j}^{s}=\frac{1}{2}\left(v_{j+e_{s}}^{s}+v_{j-e_{s}}^{s}\right), \quad s \in\{1,2,3\},
$$

where $e_{s}$ denotes the unit vector of the canonical basis of $\mathbb{R}^{3}, s=1,2,3$.

As mentionned in [2] and [8], the use of the centered discrete difference operator $D=D_{c}$ leads to conserved quantities which are not physical. On the other hand, the use of the uncentered discrete operator $\left(D=D_{+}\right.$or $D=D_{-}$) introduces some unsymmetry in the distribution function leading to a loss of accuracy. To overcome 
these difficulties, following the idea of [2] and [3], we introduce a symmetrization of the discrete operator based on the averaging of the uncentered discretizations

$$
Q_{\alpha, \beta}(f)\left(v_{j}\right)=-\frac{1}{2}\left[D_{+}^{\star}\left(\frac{1}{\left|v_{j}\right|^{3}} S\left(\tilde{v}_{j}^{+}\right) f_{j} D_{+}\left(\log f_{j}\right)\right)+D_{-}^{\star}\left(\frac{1}{\left|v_{j}\right|^{3}} S\left(\tilde{v}_{j}^{-}\right) f_{j} D_{-}\left(\log f_{j}\right)\right)\right],
$$

where

$$
\tilde{v}_{j}^{+, s}=\frac{1}{2}\left(v_{j}^{s}+v_{j+e_{s}}^{s}\right), \quad \text { and } \quad \tilde{v}_{j}^{-, s}=\frac{1}{2}\left(v_{j}^{s}+v_{j-e_{s}}^{s}\right), s=1,2,3,
$$

are chosen as in Example 3.1 to conserve the energy $\left(\tilde{v}^{+}\right.$with $D_{+}$and $\tilde{v}^{-}$with $\left.D_{-}\right)$.

We finally get the following proposition.

Proposition 3.1. The discretization (3.13) conserves total mass and decreases discrete entropy

$$
\mathcal{H}(t)=\Delta v^{3} \sum_{j \in \mathbb{Z}^{3}} f_{j}(t) \log \left(f_{j}(t)\right)
$$

Moreover, it preserves energy under the following condition on $\tilde{v}_{j}$

$$
D^{1}\left(\left|v_{j}\right|^{2}\right) / \tilde{v}_{j}^{1}=D^{2}\left(\left|v_{j}\right|^{2}\right) / \tilde{v}_{j}^{2}=D^{3}\left(\left|v_{j}\right|^{2}\right) / \tilde{v}_{j}^{3}
$$

where $\tilde{v}_{j}=\left(\tilde{v}_{j}^{1}, \tilde{v}_{j}^{2}, \tilde{v}_{j}^{3}\right)$.

Finally, if the sequence $\left(f_{j}\right)_{j \in \mathbb{Z}^{3}}$ is symmetric in all the directions (i.e. $f_{k}=f_{j}$, with $k_{s}=-j_{s}, s=1,2,3$ ), then the discrete collision operator (3.14)-(3.15) conserves the momentum.

Finally, $Q_{\alpha, \alpha}(f, f)$ is approximated in the same way as in [8]. This kind of approximation allows to verify that the discrete version of $Q_{\alpha, \alpha}(f, f)$ preserves mass, momentum and energy, and decreases the entropy. Nevertheless, this approximation remains too expensive. Hence, we use the multigrid method used in [3] to treat the computational issue.

\section{NumericAl SIMUlations}

\subsection{Linear Landau damping}

We now consider the full Fokker-Planck-Landau equation (1.1)-(1.2) where the collision operators are given by (1.3) with $\beta=\alpha$ and (2.4) with $\rho_{\beta}=1$ and $u_{\beta}=0$. The initial condition is chosen as a perturbation of the global equilibrium

$$
f_{0}(x, v)=\frac{1}{(2 \pi)^{3 / 2}} \exp \left(-|v|^{2} / 2\right)(1+A \cos (k x)), \quad(x, v) \in[0,2 \pi / k] \times \mathbb{R}^{3},
$$

where $A$ is the amplitude of the perturbation and $k$ denotes the wave number. In this subsection, $A$ is taken small enough $\left(A=10^{-5}\right)$ such that we can consider linear regimes.

To capture the Landau damping, the size of the velocity domain must be chosen greater than the phase velocity $v_{\phi}=\omega / k$ (with $\omega^{2} \approx 1+3 k^{2}$ ). Then, we set $v_{\max }=5.75$ where the velocity grid extends from $-v_{\max }$ to $v_{\max }$. We use a number of cells $N_{v}=32$ points in each direction of the velocity and $N_{x}=50$ in the one dimensional spatial direction. The boundary conditions for the distribution function are periodic in the physical space whereas the distribution function is truncated to zero for large velocities.

In this test, we are interested in the evolution of the square root of the electric energy approximated by

$$
\mathcal{E}_{h}(t)=\left(\sum_{i} \Delta x E_{i}^{2}(t)\right)^{1 / 2}
$$


Indeed, according to the Landau theory, the amplitude of $\mathcal{E}_{h}(t)$ is expected to be exponentially decreasing with a frequency $\omega$.

On Figure 1, we first represent the evolution of $\mathcal{E}_{h}(t)$ in logarithm scale, where the wave number is fixed to $k=0.3$ and different collision frequencies are taken i.e. $\nu=0,0.01$ and 0.05 . We observe that the amplitude of $\mathcal{E}_{h}(t)$ is damped exponentially in time as predicted by the Landau theory. Moreover, the influence of collisions on the electric energy is well reproduced since the increasing of the collision frequency $\nu$ induces a stronger damping rate. In conclusion, collisions play an additional role in the damping of the electric energy and its amplitude seems to be always exponentially decreasing in time.

Then, we study on Figures 2 and 3 the influence of the wave number $k$ on the evolution of the electric energy and consider $k=0.2,0.3,0.4$ and 0.5 . On the one hand, the collision frequency is set to zero. We observe that $\mathcal{E}_{h}(t)$ is always exponentially decreasing, where the damping rate is increasing when $k$ becomes larger (see Figure 2). On the other hand, we choose $\nu=0.01$ and plot the evolution of $\mathcal{E}_{h}(t)$ on Figure 3 . The behavior of the electric energy amplitude is still exponential and depends on the value of $k$ as in the previous case. Besides, we notice that the damping is more important than in the collisionless case.

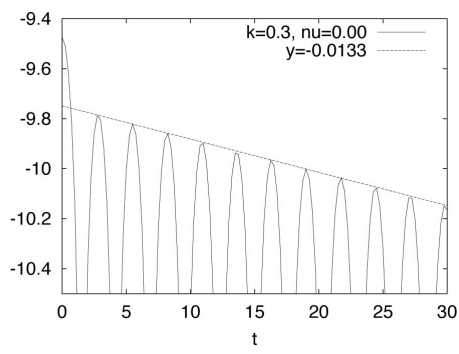

(a)

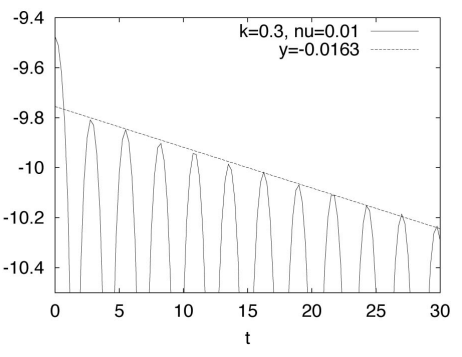

(b)

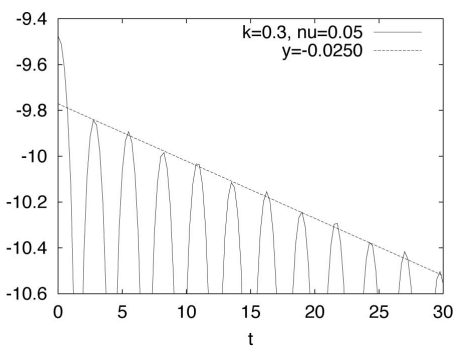

(c)

FIGURE 1. Evolution of the square root of the electric energy in $\log$ scale as a function of time: study of the influence of the collisional parameter $\nu . k=0.3$ and $\nu=0,0.01,0.05$.

The results of these tests case can be compared to estimated results. In fact, when the amplitude perturbation is small enough, the initial model can be linearized. The solution of the so-obtained linearized model is a plasma wave, the frequency and the damping of which can be computed. For the damping rate, one finds $\gamma=\gamma_{L}+\gamma_{C}$ where $\gamma_{L}$ comes from the Landau damping and $\gamma_{C}$ from collisional effects. Estimated values of $\gamma_{L}$ can be found in the literature (see $[5,9]$ ), but it is known that for small wave number $k(k \leq 0.3)$, more accurate formulas exists (see [12]). We then compare our numerical results to the following estimates

$$
\gamma_{L}=-\sqrt{\frac{\pi}{8}}\left(\frac{1}{k^{3}}-6 k\right) \exp \left(-1 /\left(2 k^{2}\right)-3 / 2-3 k^{2}-12 k^{4}\right)
$$

In the collisional case, the theoretical damping reads (see $[5,9]$ )

$$
\gamma=\gamma_{L}+\gamma_{C}, \quad \text { with } \gamma_{C}=-\frac{1}{3} \nu \sqrt{2 / \pi}
$$

where $\gamma_{L}$ is given by (4.3). In Tables 1 and 2, we compare the damping rates (4.3), (4.4) for different electrical waves in both collisional and collisionless cases, with the damping computed from our numerical method.

\subsection{Nonlinear Landau damping}

In this subsection, we also consider the Fokker-Planck-Landau equation (1.1)-(1.2) where the collision operators are given by (1.3) and (2.4) (with $\rho_{\beta}=1$ and $u_{\beta}=0$ ). The initial condition is chosen as in the previous subsection, but the perturbation $A$ is now larger. Hence, we do not consider linear regimes and the Landau 


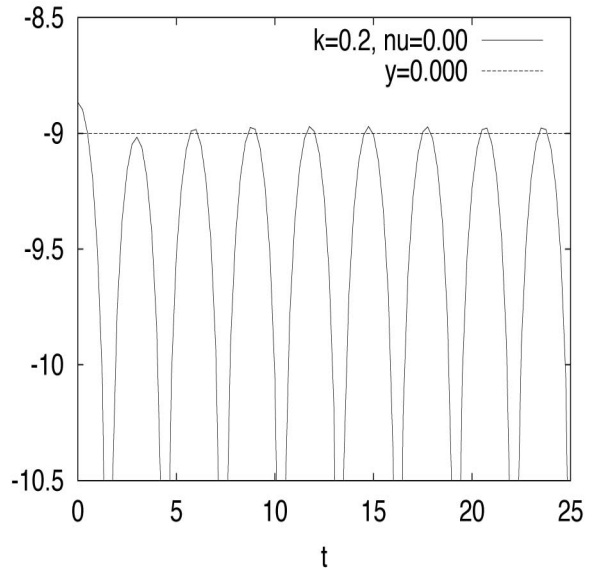

(a)

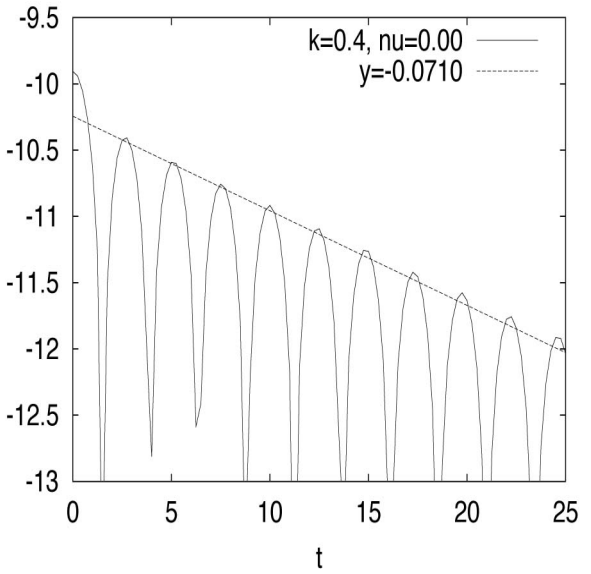

(c)

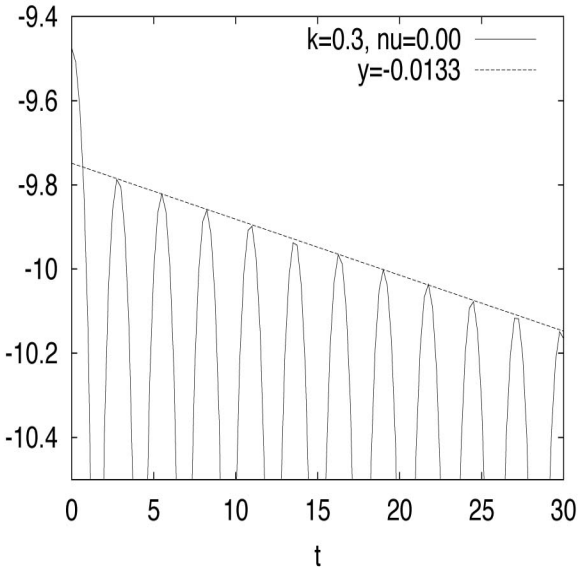

(b)

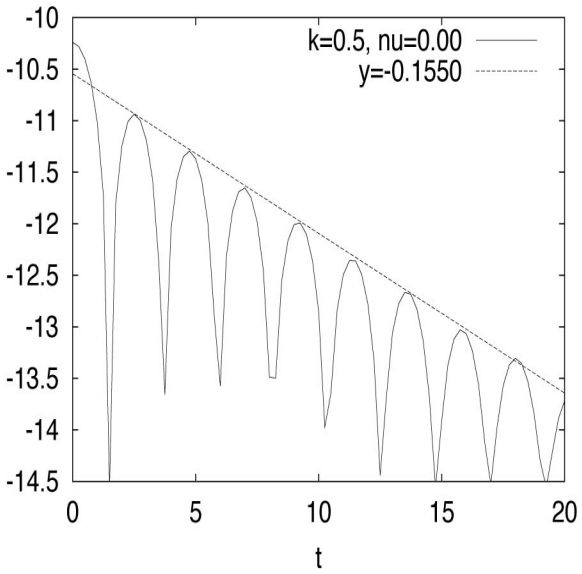

(d)

FIGURE 2. Evolution of the square root of the electric energy in $\log$ scale as a function of time: study of the influence of $k$ in the non-collisional case $(\nu=0) . k=0.2,0.3,0.4,0.5$.

\begin{tabular}{|c|c|c|c|}
\hline & Estimates $[5,9]$ & Estimates $[12]$ & Numerical \\
\hline$k=0.2$ & $-7.10^{-5}$ & $-6.10^{-5}$ & 0 \\
\hline$k=0.3$ & -0.020 & -0.0132 & -0.0133 \\
\hline$k=0.4$ & -0.096 & $\mathrm{X}$ & -0.071 \\
\hline$k=0.5$ & -0.151 & $\mathrm{X}$ & -0.155 \\
\hline
\end{tabular}

TABLE 1. Comparison of theoretical and numerical damping coefficients $\gamma$ with $\nu=0$.

theory cannot be applied. Then, we cannot compare our results to theoretical estimates but some authors already studied this test in both collisional [11] and collisionless cases [14,19].

To describe nonlinear effects, we have to consider a velocity set of size $v_{\max }=7$ in each direction. We use a number of cells $N_{v}=32$ in velocity space whereas we consider $N_{x}=50$ points in physical space. 


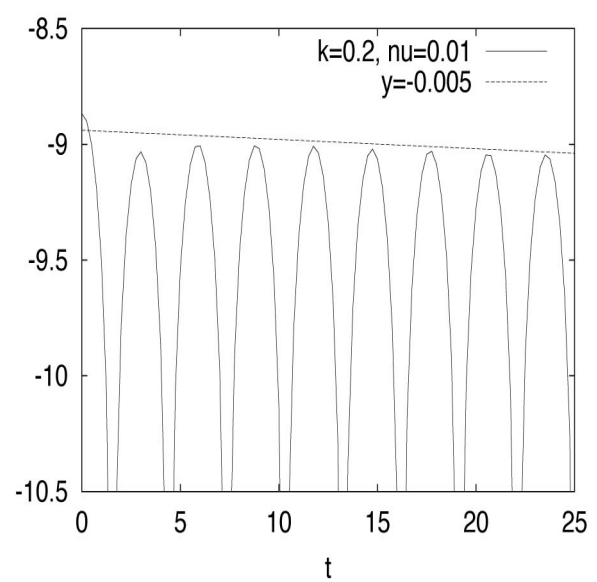

(a)

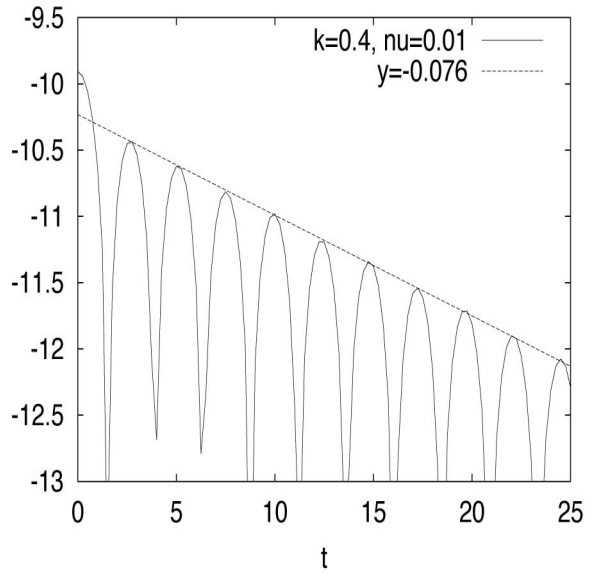

(c)

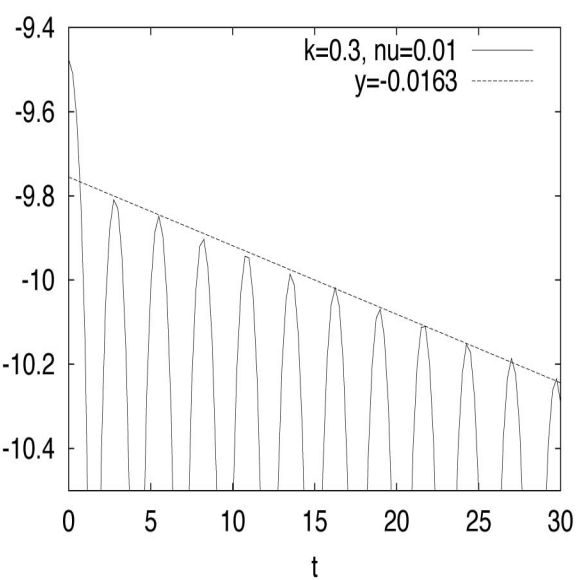

(b)

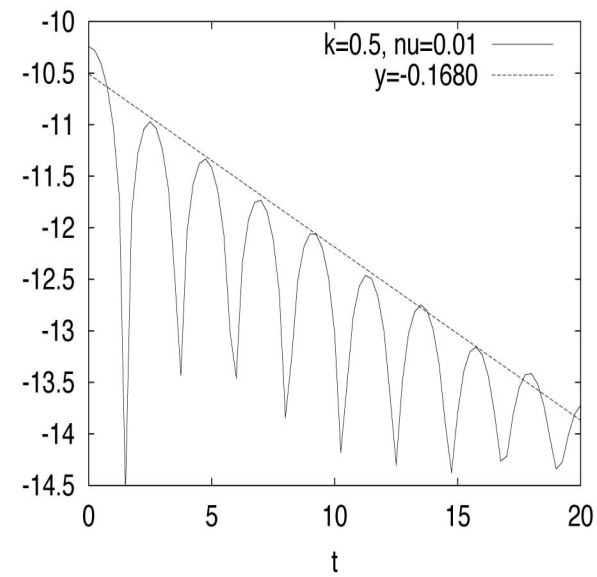

(d)

FIGURE 3. Evolution of the square root of the electric energy in log scale as a function of time: study of the influence of $k$ in the collisional case $(\nu=0.01) . k=0.2,0.3,0.4,0.5$.

\begin{tabular}{|c|c|c|c|}
\hline & Estimates [5,9] & Estimates [12] & Numerical \\
\hline$k=0.2$ & -0.003 & -0.003 & -0.004 \\
\hline$k=0.3$ & -0.023 & -0.0167 & -0.0163 \\
\hline$k=0.4$ & -0.0987 & $\mathrm{X}$ & -0.0825 \\
\hline$k=0.5$ & -0.154 & $\mathrm{X}$ & -0.168 \\
\hline
\end{tabular}

TABLE 2. Comparison of theoretical and numerical damping coefficients $\gamma$ with $\nu=0.01$.

On Figure 4, we represent the evolution of the discrete electric energy (4.2) in logarithm scale, with $A=0.2$ and $k=0.3$. Then, we compare the evolution of the electric energy for three different values $\nu=0,0.01$ and 0.05. In the collisionless regime, the results are in good agreement with the simulations presented in the literature: the electric energy is first exponentially decreasing and is next oscillating around a constant. At 
variance, in presence of collisions $(\nu \neq 0)$, these oscillations are soften. Moreover, when the parameter $\nu$ is growing up $(\nu=0.05)$, the amplitude of the electric field decreases in time, as observed in [11]. Let us pointed out that at the beginning of the simulation a damped exponential behavior is observed as in the linear case, but the damping coefficient is more important. We also plot the isovalues of the distribution function

$$
F\left(t, x, v_{x}\right)=\int_{\mathbb{R}^{2}} f\left(t, x, v_{x}, v_{y}, v_{z}\right) d v_{y} d v_{z}
$$

at time $t=0,2.5,5$ and 7.5. Without collisions, some particles are trapped around the phase velocity $v_{\phi}=\omega / k \simeq 3.84$ generating a bump, which propagates in the phase space (see [11]). However, when the collision frequency is increasing the electric energy is damped and few particles are trapped (see Figure 5).

Besides, when the global equilibrium is strongly perturbed, slope correctors act to ensure the positivity of $f$ and then the total energy is not exactly preserved, as discussed in section 3.1. Then, we study the variations of the total energy on Figure 6 for $A=0.2$. As expected, we observe that the total energy is not conserved. Nevertheless, we notice that the variations are relatively small (less than $3 \%$ ). Moreover, these variations are small compared to the variations of the total energy given by others numerical methods (semi-Lagrangian schemes).

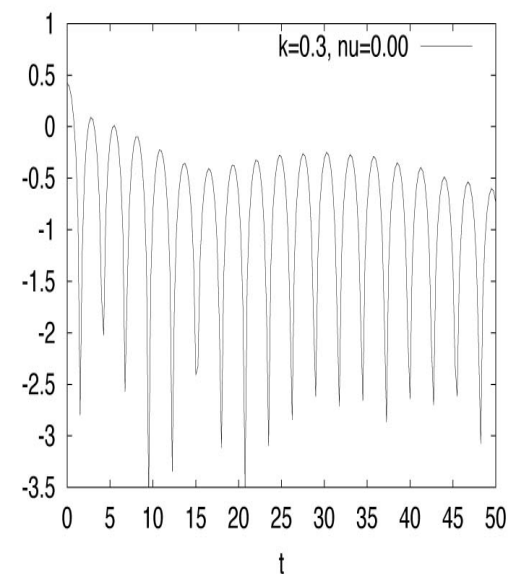

(a)

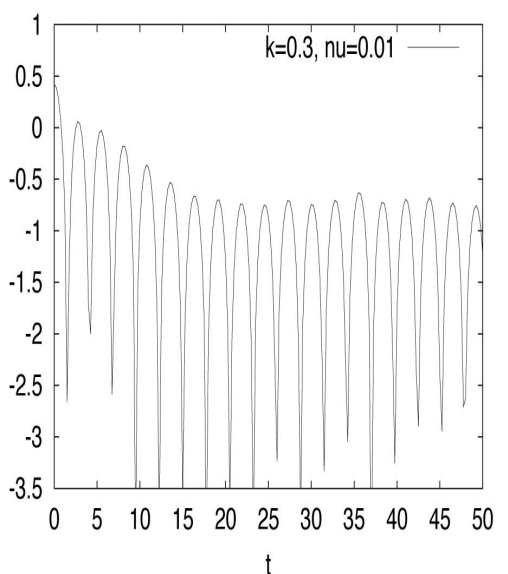

(b)

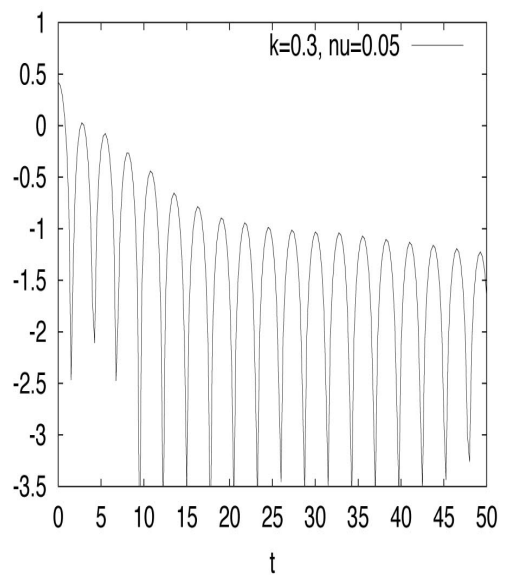

(c)

FIGURE 4. Evolution of the square root of the electric energy in $\log$ scale as a function of time: study of the influence of the collisional parameter $\nu$ in the non linear case $A=0.2, k=0.3$ with (a) $\nu=0$, (b) $\nu=0.01$ and (c) $\nu=0.05$.

\subsection{Ion acoustic waves}

The aim of this subsection is the study of the ion acoustic waves (see $[9,15,18]$ ). To that purpose, we only consider the Vlasov equation coupled with the Fokker-Planck-Landau operator (1.3) with $\alpha=\beta$ which models the ion-ion collisions. The initial condition is

$$
f_{0}(x, v)=\frac{1}{(2 \pi)^{3 / 2}} \exp \left(-|v|^{2} / 2\right)(1+A \cos (k x)), x \in[0,2 \pi / k], v \in \mathbb{R}^{3},
$$

where $A$ is the amplitude of the perturbation, and $k$ denotes the wave number. 

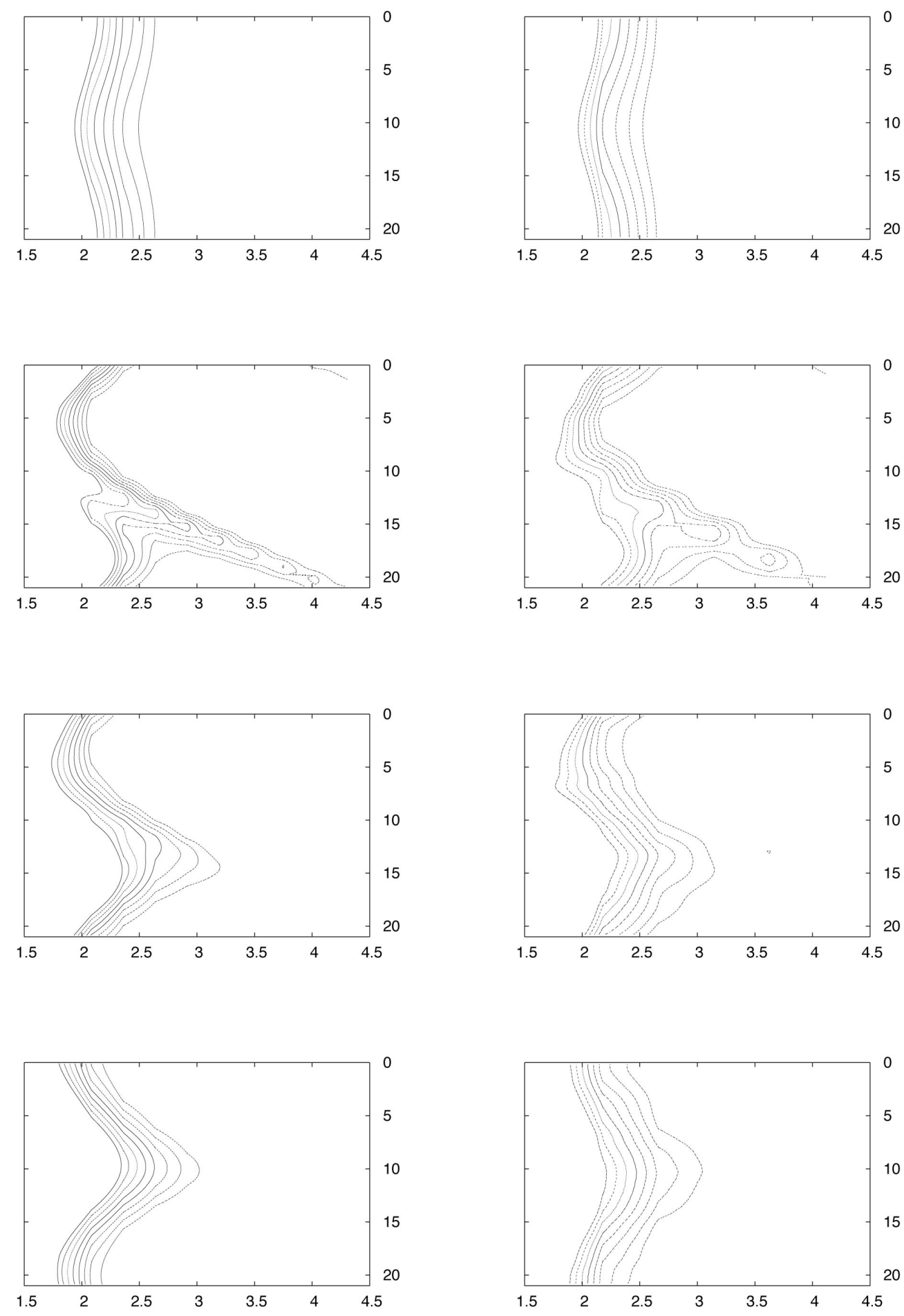

(a)

(b)

FIGURE 5. Isovalues (from 0.015 to 0.050 ) of the projection of the distribution function on the $x-v_{x}$ plane with $A=0.2, k=0.3$ and (a) $\nu=0$, (b) $\nu=0.01$. 


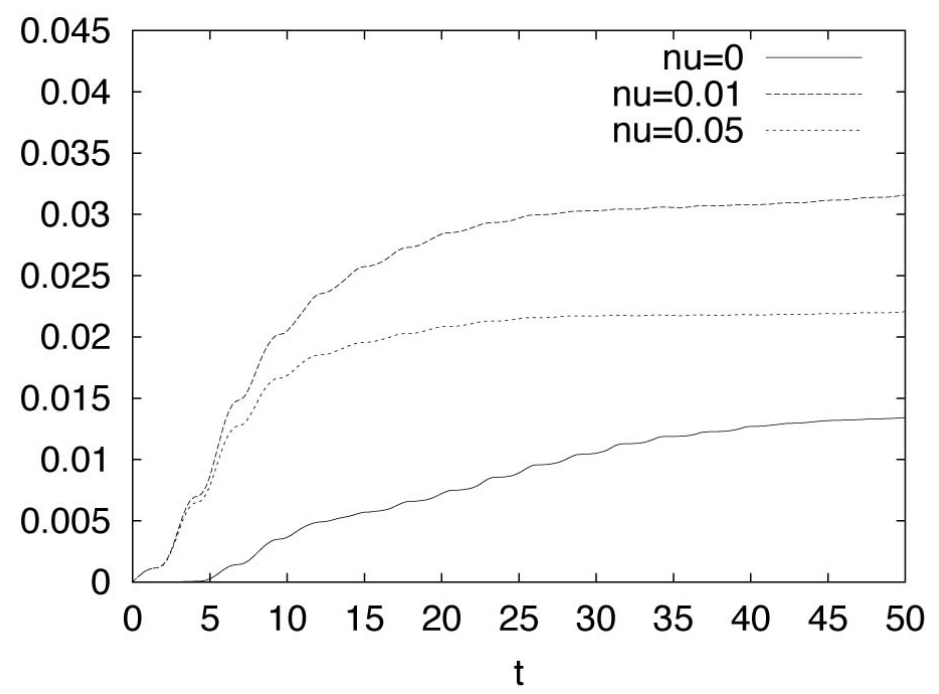

FIGURE 6. Evolution of the total energy in both collisional and collisionless case, in the non linear case $A=0.2$. $k=0.3$ with $\nu=0,0.01$ and 0.05 .

\begin{tabular}{|c|c|c|c|c|c|c|c|c|}
\hline & $\mathrm{k}=0.05$ & $\mathrm{k}=0.1$ & $\mathrm{k}=0.2$ & $\mathrm{k}=0.3$ & $\mathrm{k}=0.4$ & $\mathrm{k}=0.5$ & $\mathrm{k}=0.7$ & $\mathrm{k}=1$ \\
\hline Numerical results & 0.08 & 0.18 & 0.25 & 0.32 & 0.332 & 0.343 & 0.344 & 0.333 \\
\hline Results [15] & 0.093 & 0.165 & 0.258 & 0.331 & 0.344 & 0.342 & 0.337 & 0.33 \\
\hline Relative difference & $16.2 \%$ & $8.3 \%$ & $3.2 \%$ & $3.4 \%$ & $3.6 \%$ & $0.3 \%$ & $2 \%$ & $0.9 \%$ \\
\hline
\end{tabular}

TABLE 3. Comparison of $|\gamma| / k$ with [15]

As the ionic mean free path is bigger than the electron one, we can consider a constant electron temperature; in this case, the electric field is written

$$
E=-\frac{T_{e}}{T_{i}} \nabla_{x}(\log \rho)
$$

where $T_{e}$ and $T_{i}$ are respectively the electron and ion temperature, and $\rho$ the ion density (see [15]). We will take the ratio $T_{e} / T_{i}$ equal to $4, \nu=1$ and $k$ varies from 0.05 to 3 .

As in the previous subsection, we only consider linear regimes and take $A=10^{-3}$. The size of the velocity domain extends from $-v_{\max }$ to $v_{\max }=5.75$ and use $N_{v}=32$ grid points in each direction for the velocity grid whereas the number of spatial grid points is $N_{x}=64$. The numerical scheme presented in section 3.1 is used here. However, the electric field is not obtained through the Poisson equation in this subsection. Thus, we adapt (3.1) where $\tilde{E}_{i}^{n}$ is an approximation of (4.6) given by

$$
\tilde{E}_{i}^{n}=-\frac{T_{e}}{T_{i}} D_{c}\left(\log \rho^{n}\right)_{i}
$$

where $D_{c}$ is the usual discrete centered operator. In this case, the total energy is not conserved any more.

We are interested in the evolution of (4.2). Its amplitude is expected to be exponentially and periodically decreasing. Then, we compute numerically the damping rate $\gamma$ and the frequency $\omega$ and compare our results to the results obtained in [15]. 


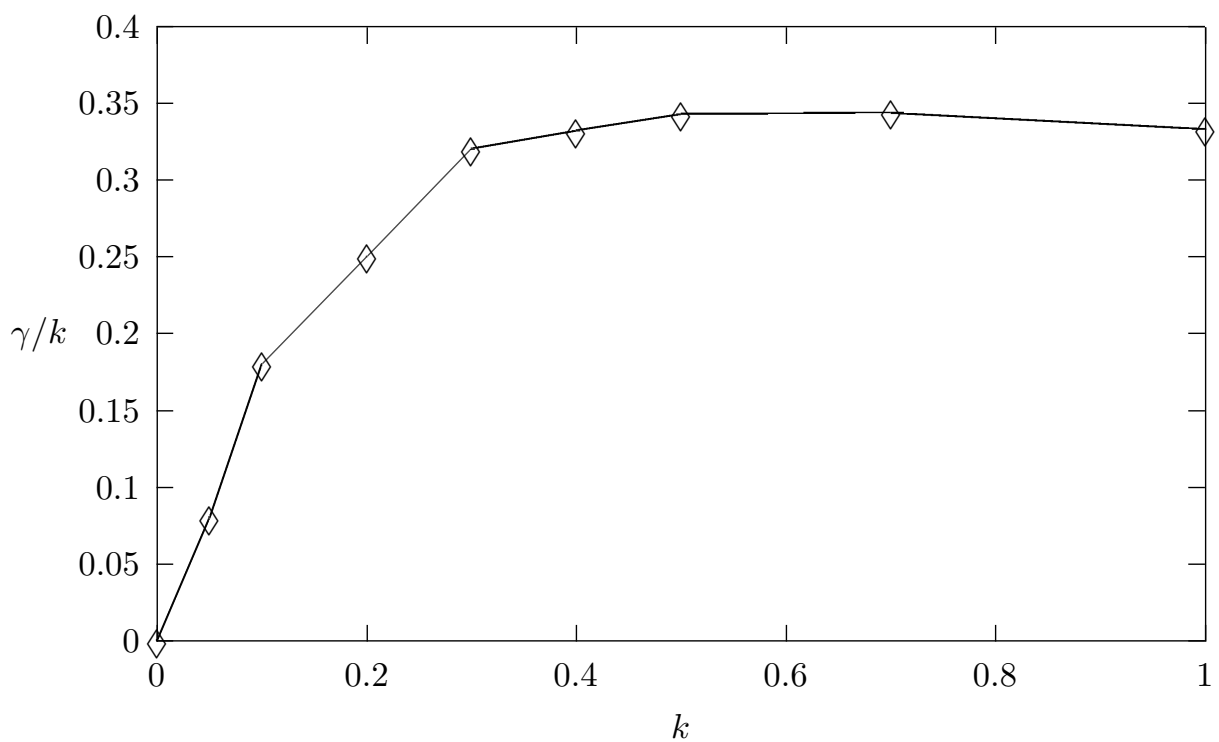

Figure 7. Study of the influence of $k$ on the damping $\gamma$.

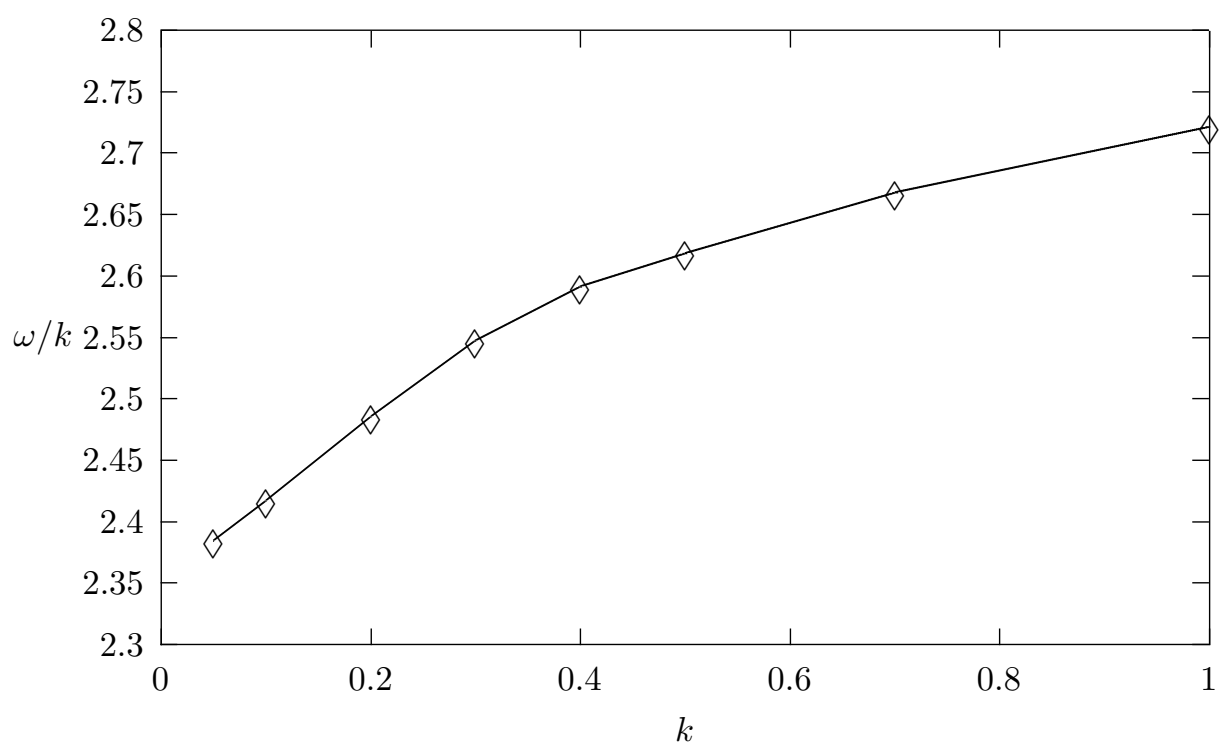

Figure 8. Study of the influence of $k$ on the frequency $\omega$. 


\begin{tabular}{|c|c|c|c|c|c|c|c|c|}
\hline & $\mathrm{k}=0.05$ & $\mathrm{k}=0.1$ & $\mathrm{k}=0.2$ & $\mathrm{k}=0.3$ & $\mathrm{k}=0.4$ & $\mathrm{k}=0.5$ & $\mathrm{k}=0.7$ & $\mathrm{k}=1$ \\
\hline Numerical results & 2.384 & 2.517 & 2.485 & 2.547 & 2.591 & 2.618 & 2.668 & 2.721 \\
\hline Results [15] & 2.38 & 2.39 & 2.42 & 2.59 & 2.66 & 2.73 & 2.76 & 2.78 \\
\hline Relative difference & $0.2 \%$ & $5 \%$ & $2.6 \%$ & $1.7 \%$ & $2.7 \%$ & $4.3 \%$ & $3.4 \%$ & $2.2 \%$ \\
\hline
\end{tabular}

TABLE 4. Comparison of $\omega / k$ with $[15]$

On Figs. 7 and 8, the quantities $|\gamma| / k$ and $\omega / k$ are plotted as a functions of $k$. One should note that the behavior of our curves is similar to [15]. Indeed, for small $k,|\gamma| / k$ is proportional to $k$, and for large values of $k$, $|\gamma| / k$ becomes stabilized and decreases slowly. Concerning the frequency, we observe that $\omega / k$ slowly increases as $k$ increases whereas in [15], $\omega / k$ becomes nearly constant for large values of $k$. From a quantitative point of view, our results for the damping rate are in good agreement with the values presented in [15], especially for large $k$ (the collisionless limit $(k \rightarrow+\infty)$ is well reached). For the frequency $\omega$, the results are also in good agreement with those obtained in [15]: the relative error is only about $4.3 \%$.

\section{Conclusion}

We have developped a new numerical scheme for realistic and collisional plasmas in one dimension in the physical space and in three dimensions in velocity space. The method takes into account two different species of particles (e.g. electrons and ions). On the one hand, a new discretization of the Vlasov-Poisson equation has been proposed. For this approximation, we proved conservation of mass and total energy. On the other hand, a discretization of the two-species collision operator is derived respecting the main properties of the continuous operator (conservation of mass, momentum, energy and decrease of the entropy). In the context of the Landau damping, our results are in a very good agreement with the theoretical results available in the literature. Furthermore, for strong perturbations we observe the effect of collisions on the damping of the electric energy and on the long time behavior of the solution. Finally, we accurately recover the results of [15] in the application of ion acoustic waves.

Several extensions and applications of this work can be considered. For instance, the ions density and mean velocity can be governed by kinetic or hydrodynamic equations coupled with the description of the electrons. Moreover, the effect of a self-consistent and applied magnetic field can be also investigated.

\section{REFERENCES}

[1] C. K. Birdsall and A. B. Langdon, Plasma Physics via Computer Simulation, Institute of Physics Publishing, Bristol and Philadelphia, (1991).

[2] C. Buet, S. Condier, Numerical analysis of conservative and entropy schemes for the Fokker-Planck-Landau equation, SIAM J. Numer. Anal., 36 (1999), 953-973.

[3] C. Buet, S. Cordier, P. Degond, M. Lemou, Fast algorithms for numerical, conservative and entropy approximations of the Fokker-Planck-Landau equation, J. Comput. Phys. 133 (1997), 310-322.

[4] C. Buet, S. Condier, F. Filbet, Comparison of Numerical schemes for Fokker-Planck-Landau equation, ESAIM Proc. 10 (1999), 161-181.

[5] F. F. Chen, Introduction to Plasma Physics and controlled fusion, 2nd edition, Plenum Press, New York and London.

[6] N. Crouseilles, F. Filbet, Numerical approximation of collisional plasmas by high order methods, J. Comput. Phys. 201 (2004), 546-572.

[7] A. De Coster, B. Perthame, P. Marcowich, Modeling of Collisions, Series in Applied Mathematics, (1998).

[8] P. Degond, B. Lucquin-Desreux, An entropy scheme for the Fokker-Planck collision operator of plasma kinetic theory, Numer. Math. 68 (1994), 239-262.

[9] J. P. Delcroix, A. Bers, Physique des plasmas, Savoirs Actuels, InterEditions, CNRS Editions, (1994).

[10] F. Filbet, E. Sonnendrücker, P. Bertrand, Conservative Numerical schemes for the Vlasov equation, J. Comput. Phys. 172 (2001), 166-187. 
[11] F. Filbet And L. PAREschi, Numerical method for the accurate solution of the Fokker-Planck-Landau equation in the non homogeneous case, J. Comput. Phys. 179 (2002), 1-26.

[12] C. J. McKinstrie, R. E. Giacone, E. A. Startsev, Accurate formulas for the Landau damping rates of electrostatic waves, Physics of Plasmas 6 (1999), 463-466.

[13] M. Lemou, Multipole expansions for the Fokker-Planck-Landau operator, Numer. Math. 78 (1998), $597-618$.

[14] G. Manfredi, Long time behaviour of the non linear Landau damping, Phys. Rev. Letters 79 (1997), 2815-2818.

[15] M. Ono, R. M. Kulsrud, Frequency and damping of ion acoustic waves, The Physics of Fluids 10 (1975), $1287-1293$.

[16] L. Pareschi, G. Russo and G. Toscani, Fast spectral methods for Fokker-Planck-Landau collision operator, J. Comput. Phys. 165 (2000), 216-236.

[17] M. Shoucri And G. KnorR, Numerical integration of the Vlasov equation, J. Comput. Phys., 14, 84-92, (1974).

[18] J. Zheng, C. X. Yu, Ion-collisional effects on ion-acoustic waves: an eigenvalue technique via moment expansion, Plasma Phys. Control. Fusion 42 (2000), 435-441.

[19] T. Zhou, Y. Guo And C.-W. Shu, Numerical study on Landau damping, Physica D 157 (2001), $322-333$. 